\title{
Controlling Halo-chaos via Wavelet-based Feedback
}

\author{
JIN-QING FANG ${ }^{\mathrm{a}, *}$, GUANRONG CHEN ${ }^{\mathrm{b}}$ and GENG ZHAO ${ }^{\mathrm{a}}$ \\ ${ }^{a}$ China Institute of Atomic Energy, P.O. Box 275-27, Beijing 102413, People's Republic of China; ${ }^{\mathrm{b}}$ Department of Electronic Engineering, City \\ University of Hong Kong, Hong Kong, People's Republic of China
}

(Received 14 May 2001)

\begin{abstract}
Halo-chaos in high-current accelerator has become one of the key issues because it can cause excessive radioactivity from the accelerators and significantly limits the applications of the new accelerators in industrial and other fields. Some general engineering methods for chaos control have been developed, but they generally are unsuccessful for halo-chaos suppression due to many technical constraints. In this article, controllability condition for beam halo-chaos is analyzed qualitatively. Then Particles-in-Cell (PIC) simulations explore the nature of beam halo-chaos formation. A nonlinear control method and wavelet function feedback controller are proposed for controlling beam halo-chaos. After control of beam halo-chaos for initial proton beam with water bag distributions, the beam halo strength factor $H$ is reduced to zero, and other statistical physical quantities of beam halo-chaos are doubly reduced. The results show that the developed methods in this paper are very effective for proton beam halo-chaos suppression. Potential application of the halo-chaos control method is finally pointed out.
\end{abstract}

Keywords: High current proton beam; Qualitative analysis; Halo-chaos; Particles in cell simulation; Nonlinear control wavelet function feedback control

PACS numbers: $\quad 05.45+b ; 41.85-p ; 29.27 b d$

\section{INTRODUCTION}

High current proton beams have attractive features for possible breakthrough applications, such as in the production of nuclear materials (e.g. tritium, transforming $\mathrm{Th}^{232}$ to $\mathrm{U}^{233}$, etc.), the transmutation of radioactive wastes, the production of radioactive isotopes for medical use and heavy ion fusion. Especially, an Energy Amplifier for cleaner and inexhaustible nuclear energy production driven by a particle beam accelerator was proposed by C. Rubbia (1993). It can promote what is beneficial and abolish what is harmful for routine nuclear energy, and makes nuclear energy system safer, cleaner and cheaper. The Energy Amplifier consists of an intense beam proton accelerator, a target and a sub-critical reactor. For example, the accelerator should provide $30 \mathrm{MW}$ beam power to drive an energy amplifier of the thermal power of $1.5 \mathrm{GW}$ with a neutron multiplying factor 0.95 . These requirements on beam power is 30 times higher than that of LAMPF, the highest one in terms of beam power in the world at present. For designing a new generation accelerator, with $10-250 \mathrm{~mA}$ of proton current at energy of $0.8-2.0 \mathrm{GeV}$ and $10-100 \%$ duty factors, and keeping beam spill below parts in $10^{-5}-10^{-8} / \mathrm{m}$, have to be satisfied in the applications. Therefore, the study of halochaos has become one of the key issues which a focusing research subject has been formulated on developing new high-power accelerators (Gluckstern, 1994; Chen and Davidson, 1994; Lagniel, 1994; Fink and Chen, 1997; Gluckstern et al., 1998; Gluckstern and Fedotov, 1999; Fedotov and Gluckstern, 1999; Wang, 1999; Fang, 2000). In this area, what needs to be resolved includes not only a better understanding mechanisms of beam halo-chaos formation but also its suppression and control. This is because beam halo-chaos can cause excessive radioactivity of an accelerator, so halo-chaos can significantly limits the applications of the new accelerators. Therefore, this subject has become a hot topic of investigation (Gluckstern, 1994; Chen and Davidson, 1994; Lagniel, 1994; Fink and Chen, 1997; Gluckstern et al., 1998; Gluckstern and Fedotov, 1999; Fedotov and Gluckstern, 1999; Wang, 1999; Fang, 2000).

Halo-chaos is essentially a turbulent motion, i.e. spatiotemporal chaotic motion within a high-power accelerator. Notice that the current research on chaos control has attracted increasing attention in several

*Corresponding author. E-mail: fjq96@ iris.ciae.ac.cn 


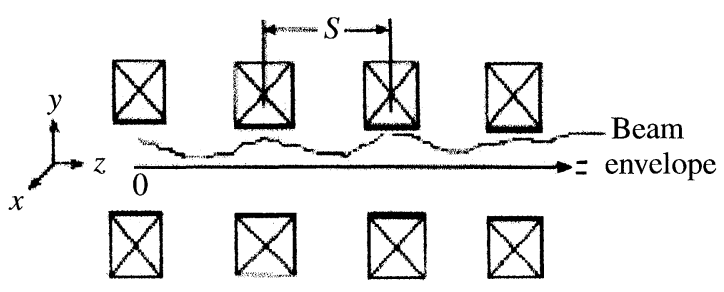

FIGURE 1 A schematic diagram of mismatched beams propagating through a periodic focusing channels.

interdisciplinary fields, where many methods of chaos control have been developed (Fang, 1996a,b; Ali and Fang, 1997; Chen and Dong, 1998; Fang and Ali, 1998; 1999; Fang et al., 1999; Fang and Chen, 2000). However, to the best of our knowledge, the issue of controlling beam halo-chaos has not been reported in the literature except for our work (Fang, 2000; Fang and Chen, 2000; Fang et al., 2001a,b). Our early attempt of controlling halochaos in new high-power accelerator has revealed some essential difficulties of this challenging subject. Yet it strongly motivates our recent study on beam halo-chaos generation mechanism and its control.

In this article, controllability condition for beam halochaos is analyzed qualitatively, and Particles-in-Cell (PIC) simulations explore the mechanism of beam halo-chaos formation. Nonlinear control methods, including wavelet function feedback controller, are proposed for controlling beam halo-chaos. After control for initial proton beam with water bag or full Gauss distributions, the beam halo strength factor $H$ is reduced to zero, and other statistical physical quantities of beam halo-chaos are doubly reduced. The PIC simulation results show that the developed methods are very effective for halo-chaos suppression. Potential application of the beam halo-chaos control method is finally discussed.

\section{QUALITATIVE ANALYSIS OF THE CONTROLLABILITY FOR HALO-CHAOS}

In view of beam halo-chaos complexity, controlling beam halo-chaos is much more difficult than other kinds of chaos. To control halo-chaos, we consider a round proton beam in a periodic focusing channel (PFC) for high current proton linear accelerator. A schematic diagram of mismatched beams propagating through the PFCs is shown in Fig. 1.

The dimensionless envelope equation of the beam propagating through the PFCs can be described by (Chen and Davidson, 1994; Gluckstern, 1994; Lagniel, 1994; Fink and Chen, 1997; Gluckstern et al., 1998)

$$
\frac{\mathrm{d}^{2} r_{\mathrm{b}}}{\mathrm{d} s^{2}}+k_{z}(S) r_{\mathrm{b}}-\frac{k}{r_{\mathrm{b}}}-\frac{1}{r_{\mathrm{b}}^{3}}=0
$$

where $r_{\mathrm{b}}$ is the beam radius and $s=z / \beta_{\mathrm{b}} c t=z / S$ the axial coordinate, in which $\beta_{\mathrm{b}} c$ is the average axial velocity of the beam particles, $c$ the light velocity and $S$ the periodicity length for a PFC. The periodic function $k_{z}(S)=k_{z}(s+S)=2 q^{2} B_{z}(s)^{2} / 4 \gamma_{\mathrm{b}} \beta_{\mathrm{b}}^{2} m^{2} c^{2}$ characterizes the strength of the focusing field, where $B_{z}(s)$ is the magnetic field on the $z$ axis, $q$ and $m$ are the particle charge and rest mass, respectively, $\gamma_{\mathrm{b}}=\left(1-\beta_{\mathrm{b}}\right)^{-1 / 2}$ is the relativistic mass factor of the beam particles. The vacuum phase advance over one axial period of such a focusing field is approximately given by $\sigma_{0}=\left[s \int_{0}^{S} k_{z}(S) \mathrm{d} S\right]^{1 / 2}=$ $\left[\eta^{2} k_{z}(0)\right]^{1 / 2}$, where $\eta$ is the tune-depression. The normalized beam preveance $k=2 q^{2} N_{\mathrm{b}} / \gamma_{\mathrm{b}}^{3} \beta_{\mathrm{b}}^{2} m c^{3}$ is a measure of the beam self-field intensity, where $N_{\mathrm{b}}$ is the number of particles per unit axial length of the beam.

A quantitative analysis on the success of the proposed nonlinear feedback controllers is rather difficult due to the complexity of the underlying envelope dynamical system (1) and its force term. Nevertheless, a qualitative analysis is given here for the completeness of the presentation of this paper.

We consider external periodic focusing field and round beam to be axis symmetric, and the proton velocity and the period of the external field are invariant in the PFCs. The control goal is to suppress the scattering of protons at radial direction. To do this, let $-g u$ be the controller used in simulations, where $u$ is either a $\sin (\cdot)$, exp (.) or a $(\cdot)^{2}$ function as shown in the list in the next section. Let also $\bar{k}_{z}$ be the mean value of the periodic function $k_{Z}(S)$ in Eq. (1). Then the controlled system is written as

$$
\frac{\mathrm{d}^{2} r_{\mathrm{b}}}{\mathrm{d} s^{2}}+\bar{k}_{z} r_{\mathrm{b}}-\frac{k}{r_{\mathrm{b}}}-\frac{1}{r_{\mathrm{b}}^{3}}=-g u
$$

In order to provide some idea as to what kind of controllers may be able to control this nonlinear system, we add a constant term $\alpha$ to both sides of Eq. (2) and then rewrite it as

$$
\frac{\mathrm{d}^{2} r_{\mathrm{b}}}{\mathrm{d} s^{2}}+\bar{k}_{z} r_{\mathrm{b}}+\alpha=\alpha-g u+\frac{k}{r_{\mathrm{b}}}+\frac{1}{r_{\mathrm{b}}^{3}}
$$

in which $\alpha$ is chosen such that the linear part on the lefthand side of Eq. (3) is stable. It is easy to verify that $\alpha>k_{z}^{2} / 4$ guarantees the stability, since the two eigenvalues of this linear part on the left hand of Eq. (3) both have negative real parts. To ensure the entire control of Eq. (3) to be stable, according to the PoincareLyapunov theorem (Verhust, 1996), a sufficient condition which is the nonlinear part of Eq. (4) must be satisfied

$$
\lim _{\left\|r_{b}\right\| \rightarrow 0} \frac{\left\|\alpha-g u+\frac{k}{r_{\mathrm{b}}}+\frac{1}{r_{\mathrm{b}}^{3}}\right\|}{\left\|r_{\mathrm{b}}\right\|}=0 .
$$

It is clear that a linear controller with the form $u=$ $u\left(r_{\mathrm{b}}\right)=a r_{\mathrm{b}}+b$ cannot satisfy Eq. (4), i.e. it is very unlikely capable of stabilizing the entire system (3) in the sense that $r_{\mathrm{b}} \rightarrow 0$ as $t_{0} \rightarrow \infty$. Therefore, generally speaking, simplest nonlinear controllers, perhaps associated with $(\cdot)^{2}, \exp (\cdot)$ and $\sin (\cdot)$, where $(\cdot)$ may be error 


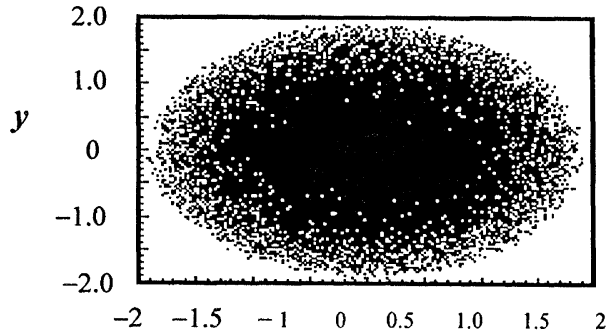

(a)

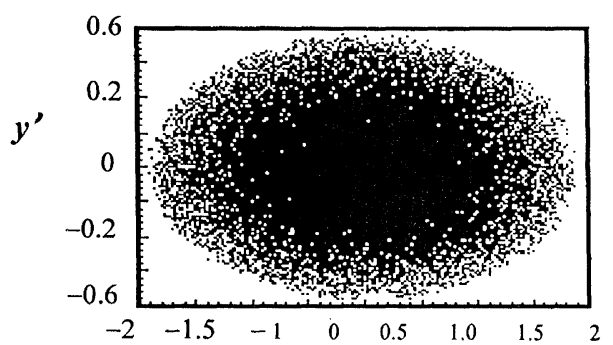

FIGURE 2 Proton beam Water-Bag gistribution. (a) The initial proton distributions in the $(X, Y)$ space and (b) the phase diagram in the $\left(X, X^{\prime}\right)$ space.

of feedback signal, such as $\left(r_{\mathrm{b}}-a_{\mathrm{m}}\right)$, are necessary, here $a_{\mathrm{m}}$ is the match radius of the beam under certain condition. It should be emphasized that the above qualitative analysis only provides some heuristic reasoning for the simulation results and observations reported in set.5. As why a quadratic or the $\exp (\cdot)$ and $\sin (\cdot)$ functions can be an effective nonlinear controller for beam halo-chaos reduction remains to be further investigated.

\section{BASIC IDEAS FOR STUDIES OF BEAM HALO- CHAOS USING PIC AND NONLINEAR FEEDBACK CONTROL}

According to basic equations of high current proton beam motion in transverse electric-magnetic field (Fang and Ali, 1999), we have developed the PIC program to simulate halo-chaos formation in the 4-dimensional phase space, in which, the particle beam self-field satisfies the Poission equation of potential:

$$
\nabla^{2} \phi(r, z)=-\frac{q}{\varepsilon_{0}} \int f\left(r, r_{\perp} ; z\right) \mathrm{d} \bar{r}_{\perp}
$$

where $\epsilon_{0}$ is the permittivity of free space, $\phi$ the potential, and $f(\cdot)$ the transverse distribution function of protons, which will be calculated in the 4-dimensional phase space.

The self-field force acted on a particle is

$$
F_{r}=-q \nabla \phi(r, z)
$$

The PIC simulation is used for studying proton motion in the PFCs. The main parameters that we calculated are as follows: the radial meshes over the length $r_{\mathrm{b}}(0)$ are 100 , total number of proton 50,0000, tune-depression $\eta=0$, mismatch factor $M=2$, the vacuum phase advance $\sigma_{0}=$ $115^{\circ}$. The calculated parameters are: match radius $a_{\mathrm{m}}=$ 0.7891642 and perveance $k=0.903079$. The evolution of 50,000 protons through the PFCs are obtained numerically using the PIC. The total energy is monitored through the PFCs since the total energy of particles is invariant in the magnetic field. The radial space-charge field of an axissymmetric beam is calculated from the Gauss law by counting the number of particles in cells of a finite radial grid, which extends up to 5 times the beam matched radius in a multi-particle simulation. A schematic diagram of proton beam propagation through PFCs is shown in Fig. 1, where the oscillatory curves illustrate the top half envelope for mismatched beam in the PFCs. Figure 2 shows the initial proton Water-Bag distributions in the $(X, Y)$ space and the phase diagram in the $\left(X, X^{\prime}\right)$ space for water bag distributions at the beginning FPCs.

In order to prevent radioactivity of the beam pipe walls and components of a high power accelerator, beam loss must be minimized. According to the qualitative analysis given above, a nonlinear feedback control strategy is proposed to achieve this control goal. The approach is to apply a nonlinear feedback controller, $G$, to the right-hand side of the particle forced Eq. (6), that is,

$$
F_{r}=-q \nabla \phi(r, z)+G
$$

According to the above analysis, the halo-chaos cannot be controlled by using a linear controller. On the other hand, nonlinear control may be realized by using either the standard Lyapunov function method or a switching manifold method, error and try, etc. (Fang, 1996a,b; Ali and Fang, 1997; Chen and Dong, 1998; Fang and Ali, 1998; 1999; Fang et al., 1999; Fang and Chen, 2000). Some typical nonlinear functions used for controlling the halo-chaos in our simulations are as follows:

$$
\left\{\begin{array}{c}
(a): G=-g \sin \left(r_{\mathrm{rms}}-a_{\mathrm{m}}\right) \\
(b): G=-g\left(r_{\mathrm{rms}}-a_{\mathrm{m}}\right)^{2} \\
(c): G=-g\left[\left(f_{a b}\left(r_{\mathrm{rms}}\right)-f_{a b}\left(a_{\mathrm{m}}\right)\right]\right. \\
\ldots
\end{array}\right.
$$

where $f_{a b}(x)$ is a special nonlinear function, so-called the wavelet-based function, $g$ the constant control gain, $r_{\mathrm{rms}}$ and $a_{\mathrm{m}}$ denote the average root-mean-square radius and the match radius of the beam envelope, respectively. In this paper, we consider an initial beam satisfying water-bag distribution. PIC simulation results have shown that the above general nonlinear control functions (GNCF) (8a) and (8b) have had preliminary suppression of halo-chaos (Fang, 2000; Fang and Chen, 2000). In this paper, it is shown that wavelet-based feedback function (8)(c) can work much well for controlling the proton beam halo-chaos. 


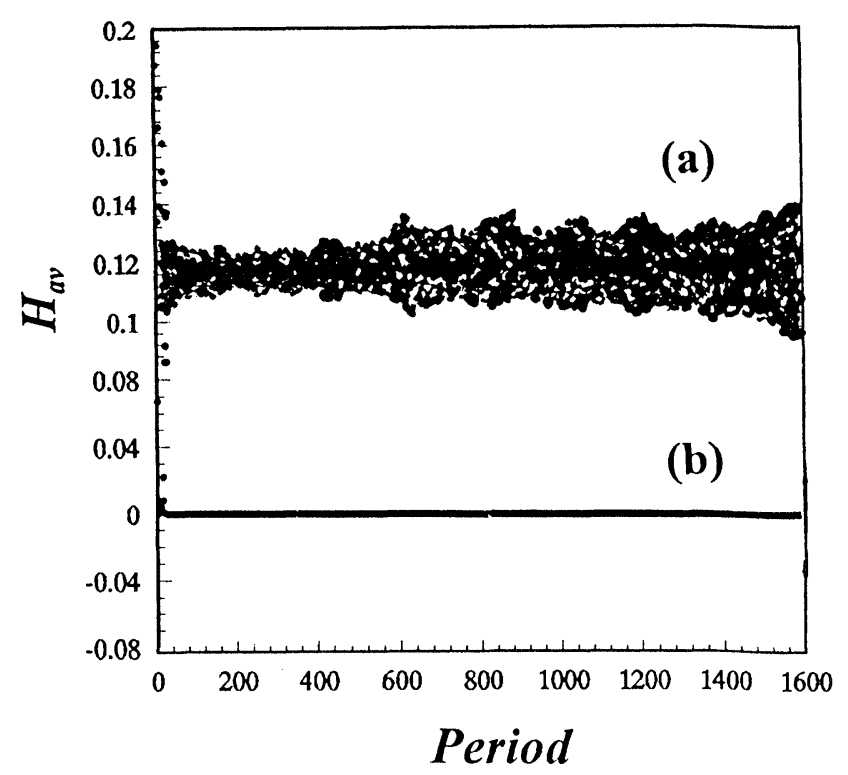

FIGURE 3 Evolution of the $H_{\mathrm{av}}$ vs numbers of the PFCs: (a) before control and (b) after control under the wavelet-based controller (10).

\section{WAVELET-BASED FEEDBACK FUNCTION CONTROL METHOD}

In this paper, we focus on the wavelet-based feedback function method, which is much better than the GNCF for control of beam halo-chaos. The mother wavelet function that we use in Eq. (8c) takes the form

$$
f_{a b}=-\frac{2}{a}\left[1-\frac{2}{a}(x-b)^{2}\right] \exp \left[-(x-b)^{2} / a\right]
$$

where $a$ and $b$ are scaling and translation constants. The main reason of using wavelet function for controller design is that it has strong nonlinearity and excellent localization property. It turns out that for halo-chaos control purpose, the translation $b$ can be very small, so for simplicity we use $b=0$ in this paper. The general form of the wavelet-based controller is taken as Eq. (8c), in which $g$ is the control gain constant, $a_{\mathrm{m}}$ is the radius of the proton beam matching, which is used as the target quantity for control. When $r_{\text {rms }} \rightarrow a_{\mathrm{m}}$ the goal of control is achieved, and in this case, it is clear that $G \rightarrow 0$, so the control action gradually vanishes.

The main reasons for choosing $r_{\text {rms }}$ for feedback control include:

i) $r_{\text {rms }}$ is a global average statistical quantity of the particle beam, i.e. the average root-mean-square radius. Whether or not the system is under control, the change of $r_{\text {rms }}$ is much stable as compared to that of the maximum radius.

ii) $r_{\text {rms }}$ reflects the spatial statistical correlation level in a particular position at a particular time.

iii) Since halo-chaos is spatio-temporat chaos, it is very sensitive to external perturbation such as mismatch factor, disturbance from the electromagnetic field and noise. Using the $r_{\text {rms }}$, insensitive to the external changes, to control halo-chaos can reduce the sensitivity to external conditional variations, thereby increasing the stability of the controlled system.

iv) Since the proton distribution function is changing according to the nonlinear and mismatched effects of spatial protons, it is natural to use statistical measurement and metod for the study of halochaos control.

Our numerical simulations reveal that beam halo-chaos can be well suppressed provided that the spatial interval period, and the control gain $g$ and control parameter $a_{\mathrm{m}}$ are chosen appropriately. We have simulated four different initial proton distributions, and designed four different controllers based on formula (9), respectively.

We discuss the control results for the initial proton water-bag distribution in this section. We used $g=9.5$, $\mathrm{b}=0$, and $a=2.0$ with wavelet feedback controller in the form of

$$
\begin{aligned}
G= & g u \\
= & -9.5\left[\left(1-r_{\mathrm{rms}}^{2}\right) \exp \left(-0.5 r_{\mathrm{rms}}^{2}\right)\right. \\
& \left.-\left(1-a_{\mathrm{m}}^{2}\right) \exp \left(-0.5 a_{\mathrm{m}}^{2}\right)\right]
\end{aligned}
$$

Using Eq. (10), we have obtained very good results for control of beam halo-chaos in the PFCs. Main results are described below.

\section{MAIN CONTROL RESULTS VIA WAVELET-BASED FEEDBACK}

\section{Changes of Statistical Average Value of Halo-chaos Strength Factor $\boldsymbol{H}_{\mathrm{av}}$ Before and After Control}

Statistical average value of beam halo-chaos strength factor, $H_{\mathrm{av}}$ is the most important characteristic quantity for measuring control result. Figure 3(a) and (b)shows the changes of the $H_{\mathrm{av}}$ with increasing numbers of the PFCs and comparison on result before and after control via the wavelet-based controller (10) applied to each period of the FPCs.

It is seen from Fig. 3 that the $H_{\text {av }}$ can be used as the most important measure of control effect for beam halochaos control. Obviously, the smaller the $H_{\mathrm{av}}$, the better the control effect. Complete control is achieved if $H_{\mathrm{av}} \rightarrow$ 0. Comparison of Fig. 3(a) with Fig. 3(b) implies that the wavelet feedback controller (10) reduced the $H_{\mathrm{av}}$ value very effectively after it was turned on. The $H_{\mathrm{av}}$ value was reduced from the maximum of 0.14 to 0.00 . This means halo-chaos is completely suppressed.

\section{Comparison of Evolution of Maximum Proton Beam Radius}

Figure 4(a) and (b) gives the evolution of maximum proton beam radius $r_{\max }$ before and after the wavelet-based 


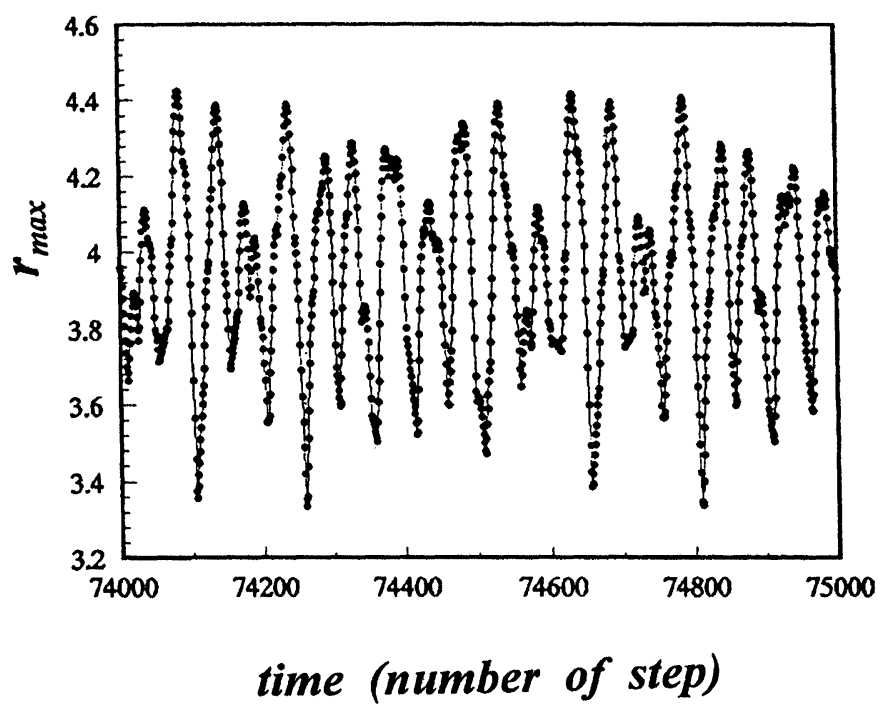

(a)

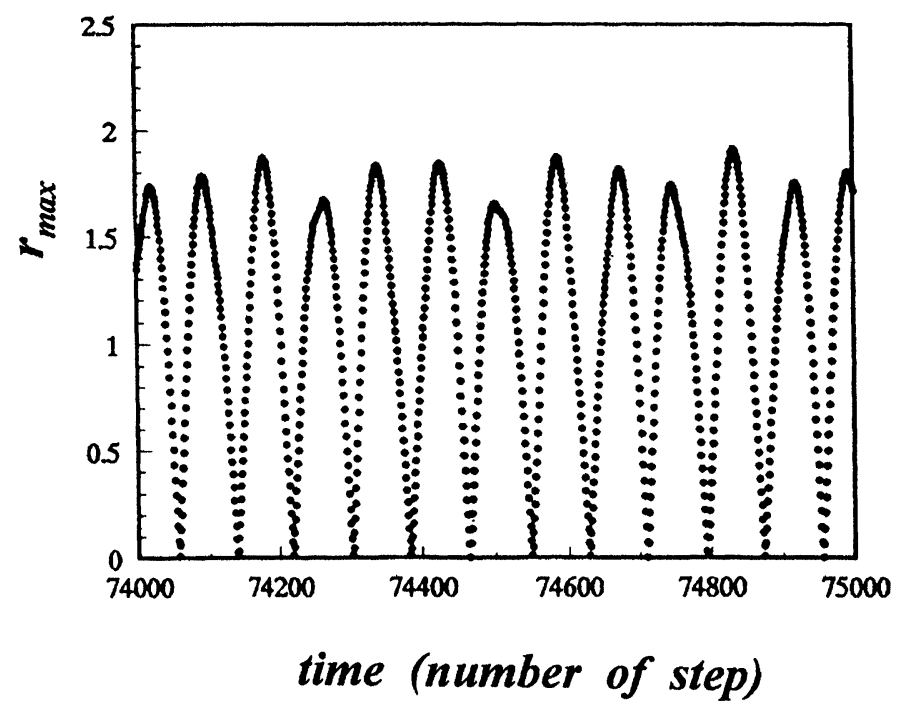

(b)

FIGURE 4 Evolution of maximum proton beam radius $r_{\text {max }}$ : (a) before control; (b) after control.

feedback controller (10) was applied to each period of the FPCs.

It is seen from Fig. 4 that the maximum radius of proton beam, $r_{\max }$, changes rapidly without control, which indeed was chaotic. It became relatively stable with very small magnitude, albeit showing little irregularity still after control. From the statistical point of view, the radius of its envelope has been significantly reduced by $2-3$ times under the control of Eq. (10). And $r_{\max } / a_{\mathrm{m}}$ is reduced about 4-5 times after control (10) was turned on at the $P=1200$.

\section{Comparison of Evolution of Mean Root-square Radius $\boldsymbol{r}_{\max }$ of Proton Beam}

Figure 5(a) and (b) shows the evolution of mean rootsquare radius $r_{\text {rms }}$ of proton beam before and after the wavelet-based feedback controller (10) was applied to each period of the FPCs.

It is clear from the Fig. 5 that the evolution of rms radius of proton beam, $r_{\text {rms }}$, chaotic, irregular, and scattered around with large radii before control, and yet it became almost quasi-periodic around the beam core with very 


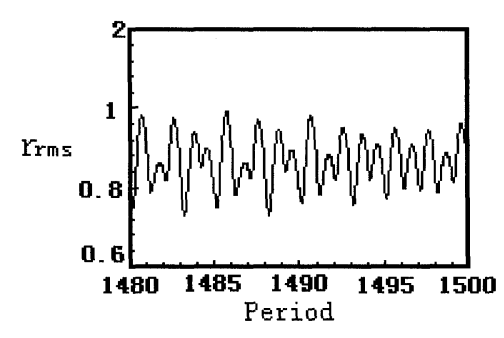

(a)

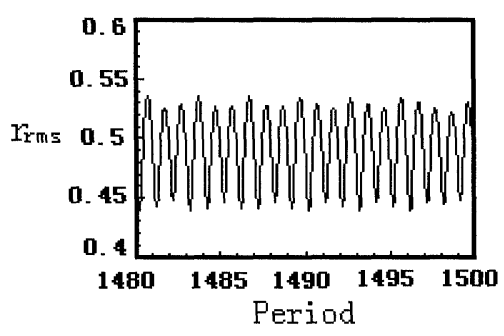

(b)

FIGURE 5 Evolution of mean root-square radius $r_{\mathrm{rms}}$ of proton beam: (a) before control; (b) after control.

small magnitudes (reduced to maximum 0.53) after feedback (10) was applied. The $r_{\text {rms }}$ is significantly reduced by nearly 2 times. Therefore, it implies that beam halo-chaos is suppressed effectively although it is still in chaos.

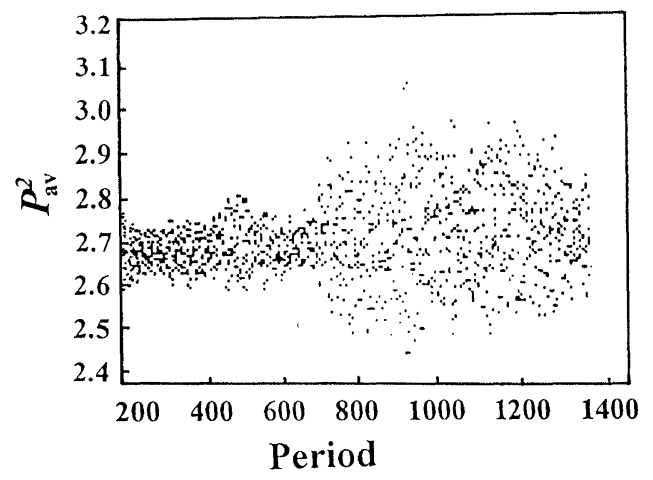

(a)

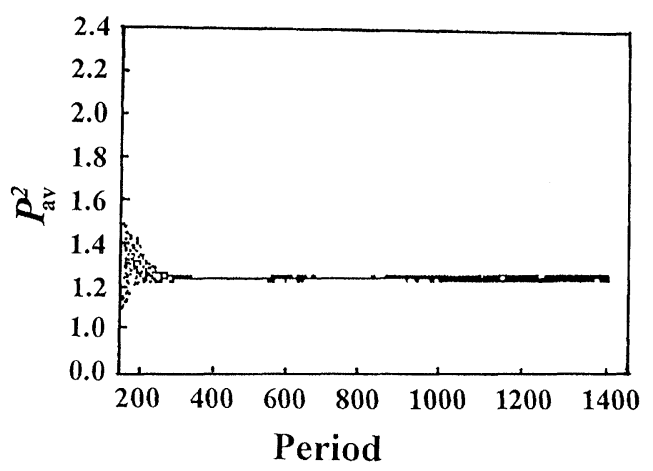

(b)

FIGURE 6 Evolution results of mean-square momentum $p_{\text {av }}$ of proton beam: (a) before control; (b) after control.
Comparison of Evolution of Transverse Mean-square Momentum $p_{\text {av }}$ of Proton Beam

Figure 6(a) and (b) are the evolution results of transverse mean-square momentum, $p_{\mathrm{av}}^{2}$, of proton beam before and after controller (10) was applied to each period of the FPCs.

It is clear from the Fig. 6 that the proton beam was scattered around with large radii due to the transverse mean-square momentum $p_{\mathrm{av}}^{2}$, is very large (about maximum 2.2) before control, and yet it became stable around the beam core with very small radii because of largely reduced $p_{\mathrm{av}}^{2}$ to about 1.2 after the wavelet feedback (10) was applied. The essential reason for that is that the transverse energy of proton beam is reduced doubly. This control result is consistent with the minimum energy control principle we proposed in Luo et al. (1999).

\section{Comparison of Evolution of Relative Emittance of Proton Beam}

Figure 7(a) and (b) are the evolution results of average relative emittance $\epsilon_{x}$ of proton beam before and after the wavelet-based feedback controller (10) was applied to each period of the FPCs.

Emittance is also an important quantity for measuring beam transport in the accelerators. The smaller the it is, the better will it be. For any distribution in the phase space, emittance can be described by an rms ellipsoid. The projection of the emittance on the $x-P_{x}$ plane, $\epsilon_{x}$ is defined by $\varepsilon_{x}=\sqrt{x^{2} p_{x}^{2}-\overline{x p_{x}}} / m c$. Similarly, $\epsilon_{y}$ can also be defined on $y-P_{y}$ plane.

It is clear from Fig. 7 that the $\epsilon_{x}$ of proton beam has been largely reduced from 2.8 to 0.99 after the wavelet feedback control (10).

\section{Comparison of Phase Portraits of Halo-chaos Before and After Control}

Figures 8 and 9 show the phase diagrams of proton beam halo-chaos in the $(X, Y)$ space before and after applied the wavelet-based feedback control (10), respectively.

It is seen from the comparison of Fig. 8 with Fig. 2(a) that the proton distribution area of halo chaos is increased from 12.56 to 28.26 , it is nearly expended by more than 2.5 times before control at $P=1200$. But after control was applied to the FPCs periodically, such as it has been reduced from 19.6 to 6.2 .3 at $P=300$ by about 3.1 times after comparing Fig. 8(a) with Fig. 9(a). At $P=1200$ it is reduced from 28.3 to 3.15 , nearly reduced by about 9 times after comparing Fig. 8(d) with Fig. 9(d). These results means that the resulting area of proton distribution is much less than area of the initial distribution at Fig. 2(a). In other words, the radius of proton halo-chaos are contracted from 2.5 to less than 1 . Thus halo-chaos is naturally suppressed.

Figures 10 and 11 shows the phase diagrams of proton beam halo-chaos in the $\left(X, X^{\prime}\right)$ space before and after the applied wavelet-based feedback control (10), respectively. 

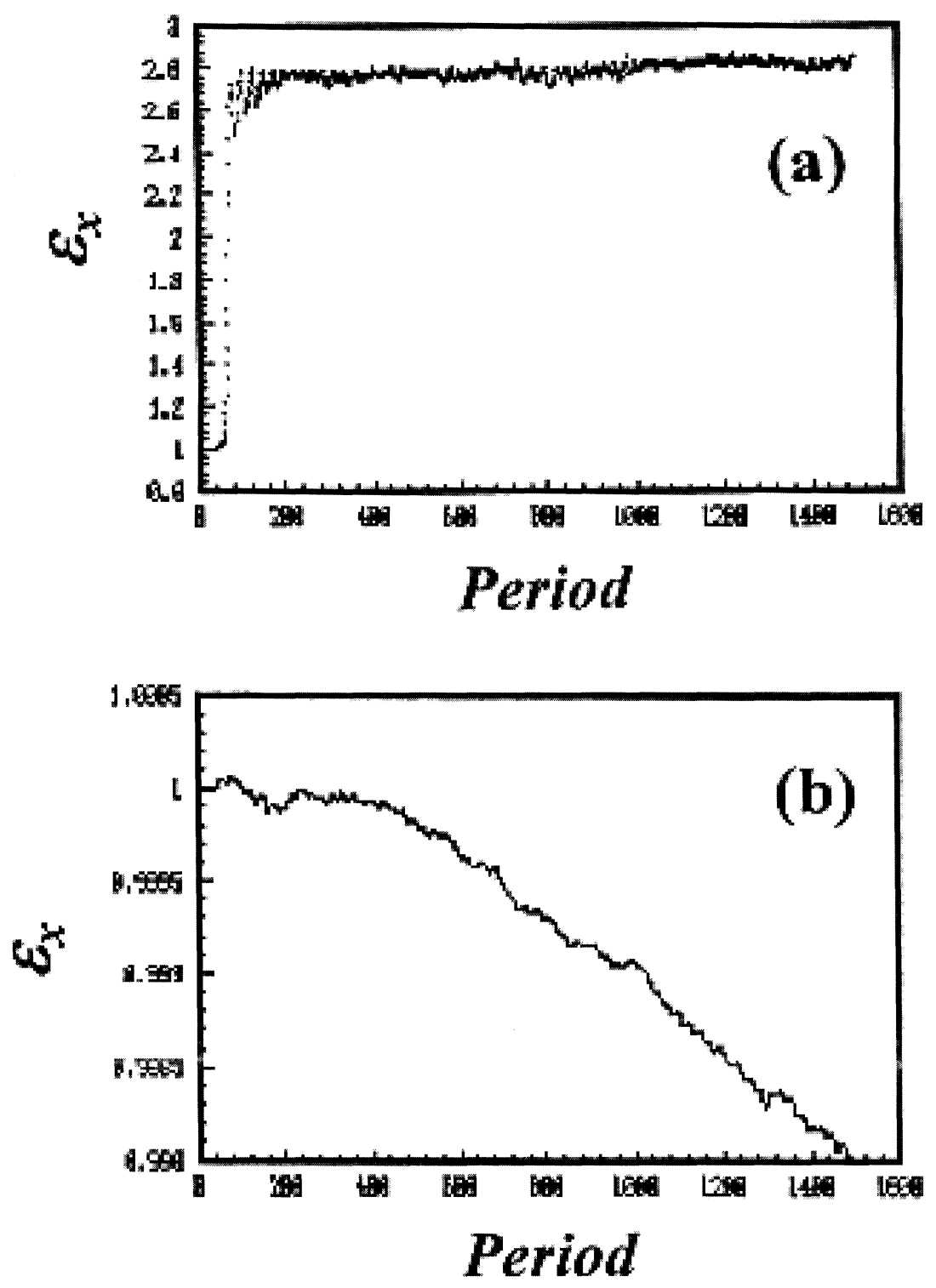

FIGURE 7 Evolution results of average emittance of proton beam: (a) before control; (b) after control.

Similarly, it is clear from comparison of Fig. 10 with Fig. 11 that the proton phase diagrams, not only their area, are largely reduced, for example at $P=300$ it is reduced from about 28 to less than 10 , nearly contracted by about 3 times, but also maximum velocities of protons are doubly decreased from 6 to less than 3 , as well as their shapes of phase portraits are spiraling when control is going on at different focusing periods. Density of proton-core is almost focused on the axis center.

All results above imply the control effect of the waveletbased feedback is extremely well for suppressing beam halo-chaos in the FPCs.

\section{DIFFERENT KINDS OF WAVELET FEEDBACK FUNCTIONS FOR DIFFERENT INITIAL PROTON DISTRIBUTIONS}

It should be emphasized that we can design the different kinds of wavelet feedback controller to achieve effective control for different initial proton distributions, such as the Kapchinsky-Vladimirsky (K-V), full Gauss and $3 \sigma$ Gauss distributions. These wavelet feedback controllers are as follows:

(1) Initial protons obey the K-V distribution:

In this case, we used $g=9.5, a=2.8$ and $b=0$ with wavelet feedback controller in the form of

$$
\begin{aligned}
G= & g u \\
= & -9.5 \\
& \times \frac{2}{2.8}\left[\left(1-\frac{2}{2.8} r_{\text {rms }}^{2}\right) \exp \left(-\frac{2}{2.8} r_{\text {rms }}^{2}\right)\right. \\
& \left.-\left(1-\frac{2}{2.8} a_{m}^{2}\right)\right]
\end{aligned}
$$


The Period---300

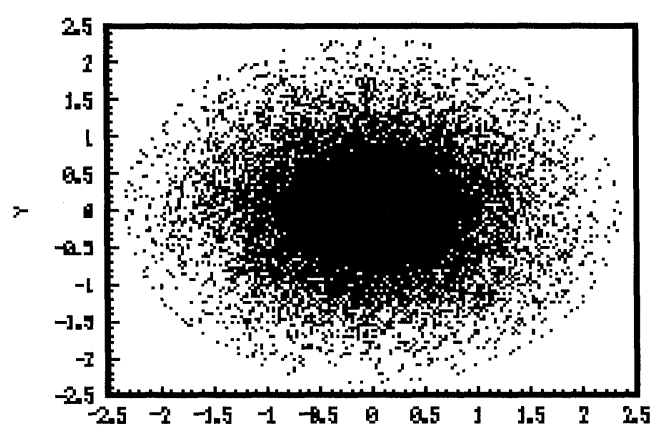

The Period---900

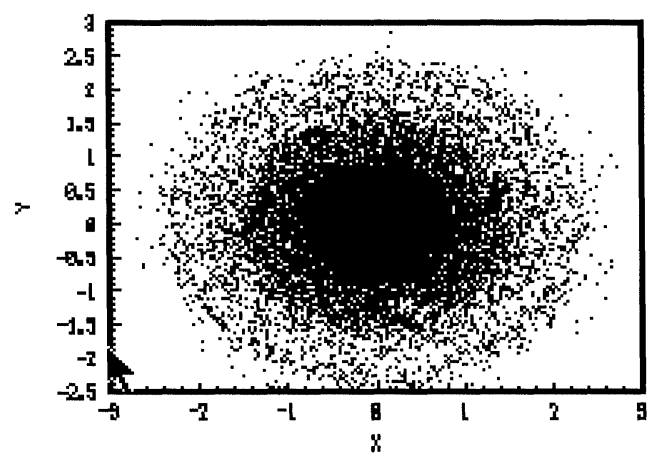

The Period--- 600

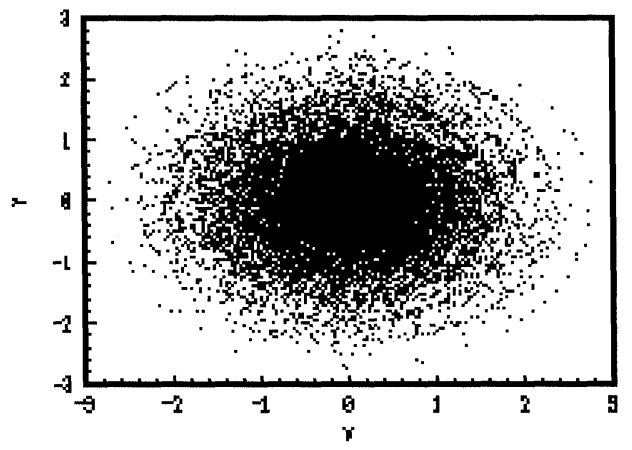

The Period-----1200

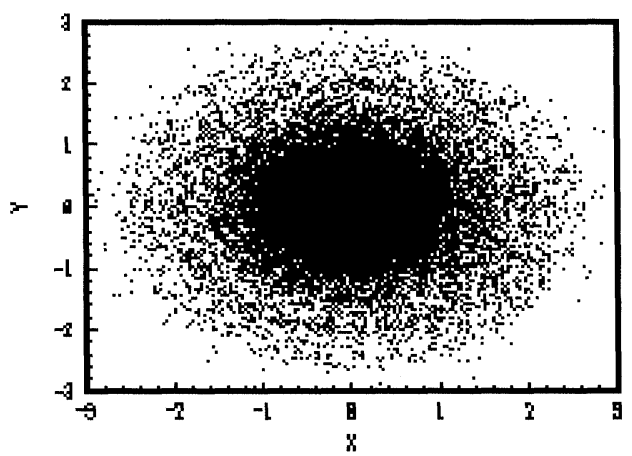

FIGURE 8 Proton distribution diagram in the $(X, Y)$ space at different periodic section before control: (a) $P=300$; (b) $P=600$; (c) $P=900$; and (d) $P=1200$.

(2) Initial protons obey the $3-\sigma$ Gauss distribution:

In this case, we used $g=9.5, a=2.0$ and $b=0$ with wavelet feedback controller in the form of

$$
\begin{aligned}
G= & g u \\
= & -9.5\left[\left(1-r_{\mathrm{rms}}^{2}\right) \exp \left(-0.5 r_{\mathrm{rms}}^{2}\right)-(1\right. \\
& \left.\left.-a_{\mathrm{m}}^{2}\right) \exp \left(-0.5 a_{\mathrm{m}}^{2}\right)\right]
\end{aligned}
$$

(3) Initial protons obey the complete Gauss distribution:

In this case, we used $g=9.5, a=0.7$ and $b=0$ with wavelet feedback controller in the form of

$$
\begin{aligned}
G= & g u \\
= & -9.5 \\
& \times \frac{2}{0.7}\left[\left(1-\frac{2}{0.7} r_{\mathrm{rms}}^{2}\right) \exp \left(-\frac{1}{0.7} r_{\mathrm{rms}}^{2}\right)\right. \\
& \left.-\left(1-\frac{2}{0.7} a_{\mathrm{m}}^{2}\right) \exp \left(-\frac{1}{0.7} a_{\mathrm{m}}^{2}\right)\right]
\end{aligned}
$$

The results of halo-chaos characteristics before and after control under the above wavelet-based controllers are summed up in Table I, respectively.
It can be seen from Table I that

1. Halo-chaos strength factor, $H$, can be used as the measure of control effect for halo-chaos control. It is the number of particles located outside the radius $r_{\mathrm{b}}=$ $1.75 r_{\mathrm{b}}(0)$ of the envelope of the beam, versus the number of total particles used in the simulation. Obviously, the smaller the $H$, the better the control effect. Complete control is achieved if $H \rightarrow 0$. Table I shows that the wavelet feedback controllers reduce the $H$ value very effectively: after the controller was turned on at the 1000th periods, the $H$ value decreases from $0.43(\max )$ or $0.28(\mathrm{~min})$ to 0.00 . This means halo-chaos is completely suppressed. It should be pointed out that $H \rightarrow 0$ and good results can be reached even after about a thousand periods at the FPCs while the wavelet feedback controller has been periodically applied to the FPCs.

2. The root-square momentum of the protons can be significantly reduced by $2-3$ times. So transverse energy of protons is also much reduced correspondingly. Moreover, $r_{\max } / a_{\mathrm{m}}$ is reduced 2-3 times after controller was turned on at the 1000th periods.

3. The radius of protons, in both $x$ - and $y$-directions, can be significantly reduced. It is decreased by about $2-3$ times after control. 
The period-- 300

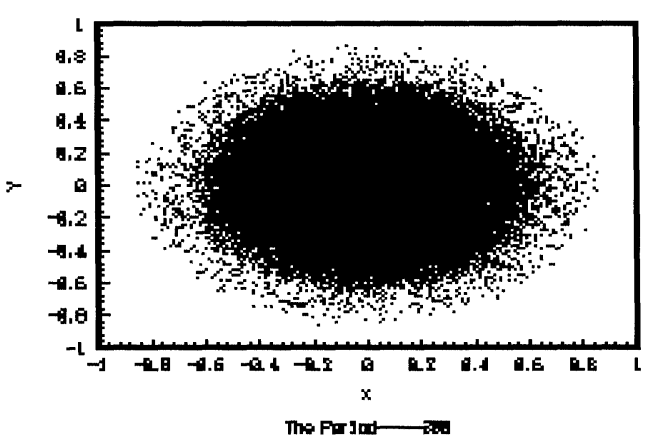

The Feriod---900

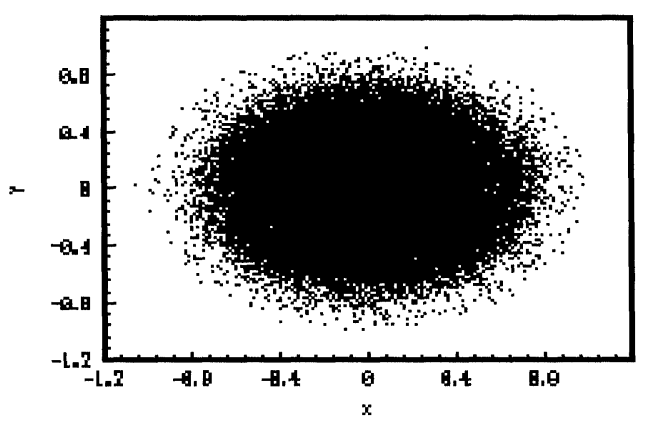

The period-- 600

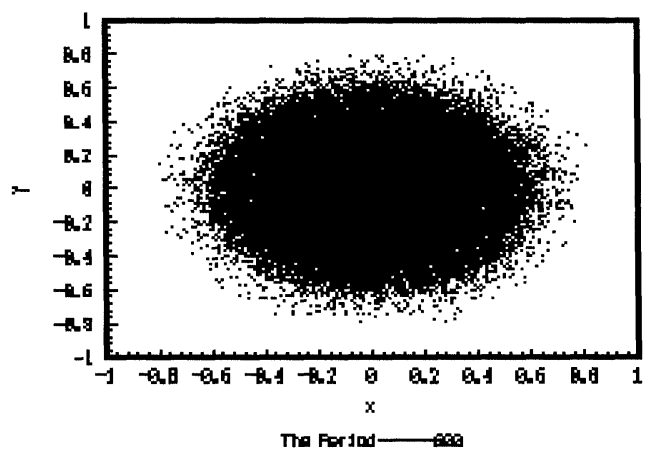

The Period---1200

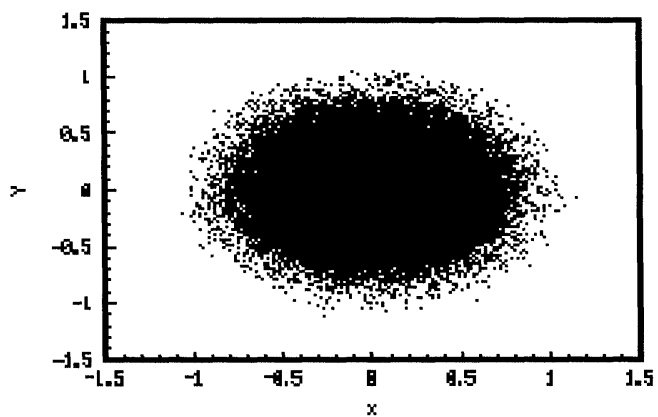

FIGURE 9 Phase diagram in the $(X, Y)$ space corresponding to Fig. 8 after control.

4. After control is applied, almost all statistical properties of the halo-chaos are in good condition, which are demonstrated in the section "Discussions and conclusions".

In summary, the wavelet-based feedback controller performs very well on halo-chaos suppression control.

\section{DISCUSSIONS AND CONCLUSIONS}

In this paper, we has proposed and verified wavelets feedback control method based on nonlinear control for controlling halo-chaos of proton beam with four kinds of different initial proton beam distributions, mainly the Water-Bag initial proton distribution. The PIC has verified that this control methodology is very effective for suppressing beam halo-chaos in high-current proton beam transport in the PFCs. Under the control, almost all statistical properties of the halo-chaos are in good condition, such as, the halo-chaos strength factor becomes zero, mean-square beam radius and mean-square beam momentum or transverse energy as well as relative emittance are effectively reduced by 2-3 times. Both theoretical and numerical results show that the proposed wavelet feedback function as a special nonlinear function, is efficient and has attractive potential application future.

As we have revealed some reasons of beam halo-chaos formation in Fang (2000), Fang and Chen (2000) and Fang et al. (2001a,b), our results show again that the halo-chaos formation depends not only on the nonlinear space charged effect but also on the interaction between the particles and the particle-core, which leads to transverse energy exchange. It also confirms that resonance overlapping forms stochastic areas and "phase" mixing diffusion takes place in these stochastic areas (Fang, 2000; Fang and Chen, 2000; Fang et al., 2001a,b). The physical mechanism using this kind of controls is also revealed to be the fact that the nonlinear feedback forces counteract the nonlinear effect of the beam space charge and reduce transverse energy of proton beam since the mean-square momentum of proton beam is reduced largely. In other words, the nonlinear feedback forces can suppresses the scattering of the transverse particles with higher energy.

The control methods proposed in this article, we think, should be useful to design new generation accelerator and is also easily tested in experiments. For example, external radio frequency fields can be coupled into the PFCs periodically and sporadically, and the parameters can also be adjusted in a right way to achieve the goal of control (Fang and Chen, 2000). Moreover, wavelet technique has been applied to many industries and experiments (Chui, 1997). Of course, this subject on controlling beam halochaos as complexity has just been in research beginning stage. A more effective nonlinear feedback control approach along the same line will be further developed for much more practice applications, hopefully with more rigorous quantitative analysis of mechanism for 
The Period---300

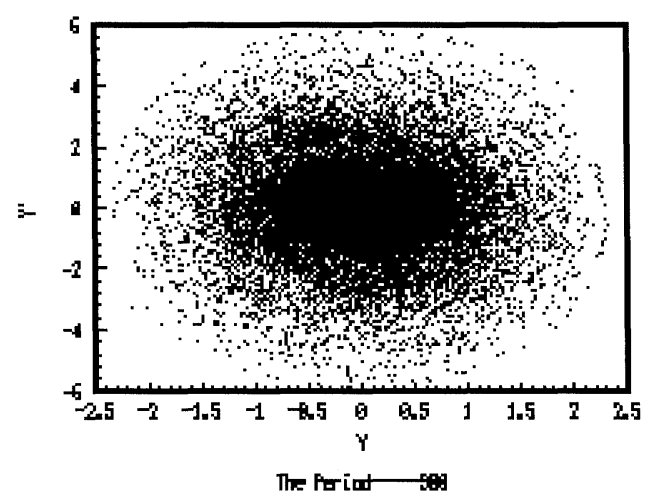

The Period---900

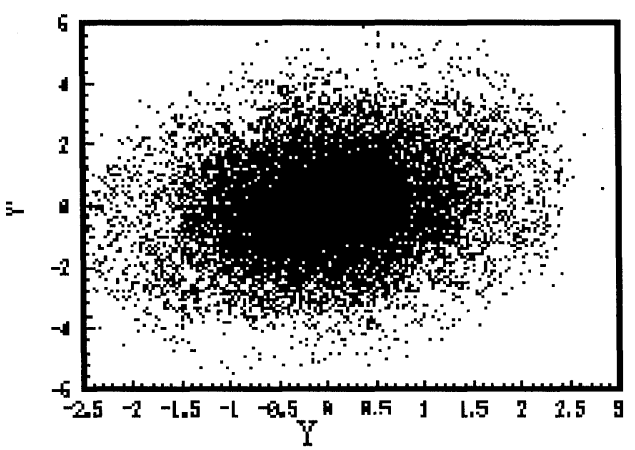

The Period---600

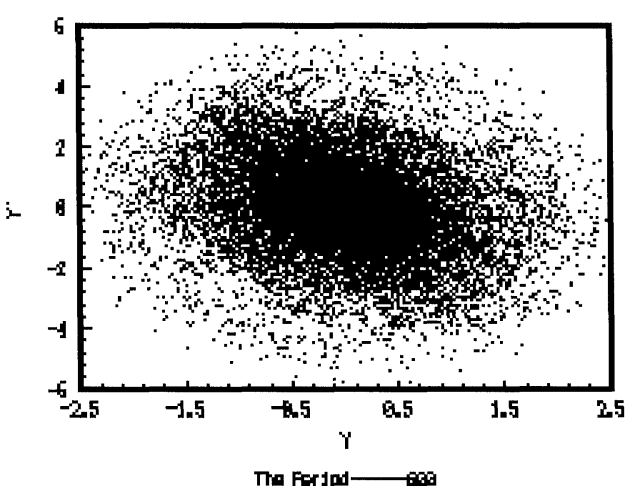

The Period----1200

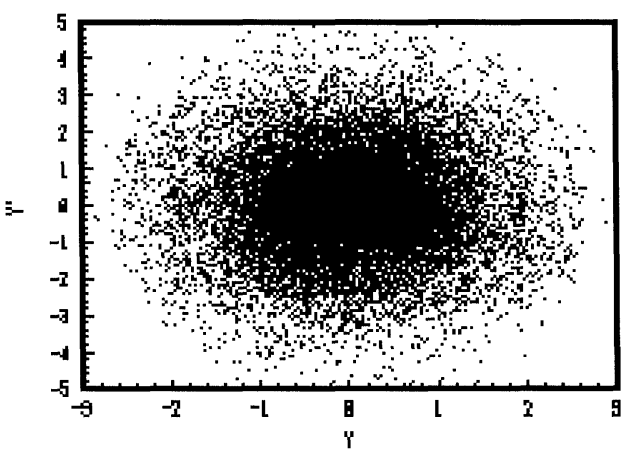

FIGURE 10 Phase diagram in the $\left(X, X^{\prime}\right)$ space at different periodic section before control: (a) $P=300$; (b) $P=600$; (c) $P=900$; and (d) $P=1200$.

The Period---300

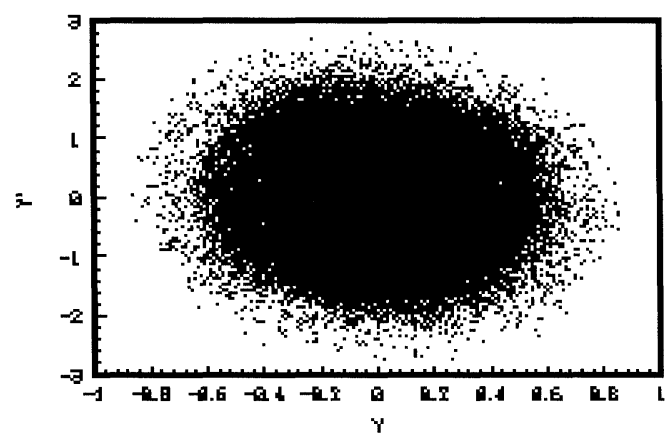

The Period---900

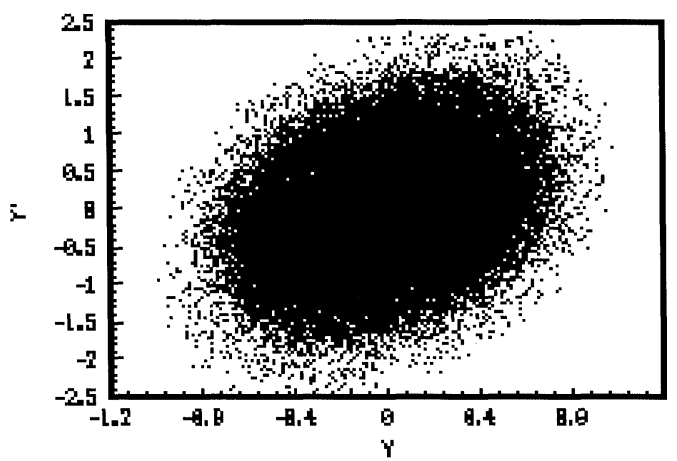

The Period---600

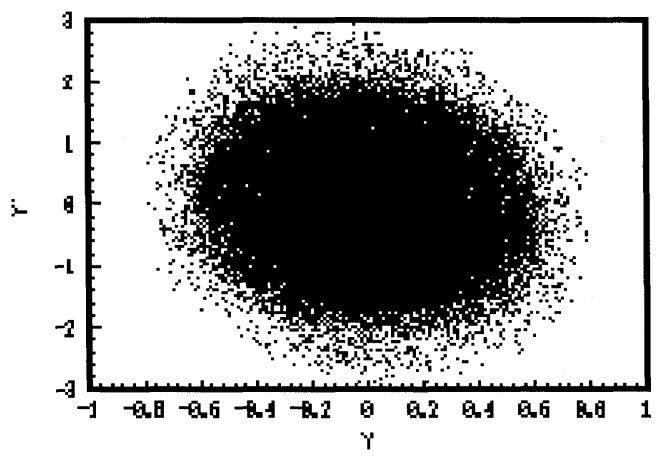

The Period---1200

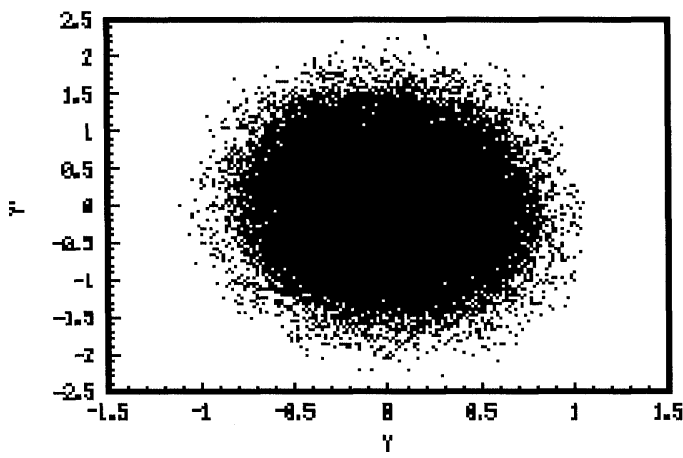

FIGURE 11 Phase diagram in the $\left(X, X^{\prime}\right)$ space corresponding to Fig. 10 after control. 
TABLE I Comparison of halo-chaos characteristics before control with after four wavelet feedback controllers applied to the FPCs periodically

\begin{tabular}{lcccc}
\hline $\begin{array}{l}\text { Initial proton distribution and } \\
\text { wavelet feedback controller }\end{array}$ & $\begin{array}{c}H \\
\text { (before/after control } \\
\text { PFC }=1000)\end{array}$ & $\begin{array}{c}r_{\text {max }} / a_{\mathrm{m}} \\
\text { (before/after control } \\
\text { PFC = 1000) }\end{array}$ & $\begin{array}{c}\text { Radiation } \epsilon_{x}, \epsilon_{y} \\
\text { (before/after control } \\
\text { PFC = 1000) }\end{array}$ & $\begin{array}{c}\text { Momentum root-square } p_{\mathrm{av}}^{2} \\
\text { (before/after control } \\
\text { PFC }=1000)\end{array}$ \\
\hline K-V (11) & $0.43 / 0.00$ & $5.20 / 2.10$ & $2.88 / 1.00$ & $3.62 / 1.31$ \\
3- $\sigma$ Gauss (12) & $0.28 / 0.00$ & $4.32 / 1.31$ & $2.89 / 1.11$ & $3.09 / 1.61$ \\
Gauss (13) & $0.31 / 0.00$ & $4.95 / 1.40$ & $2.91 / 1.04$ & $3.68 / 2.94$ \\
Water-bag (10) & $0.298 / 0.0$ & $4.24 / 2.00$ & $2.87 / 1.25$ & $2.58 / 1.40$ \\
\hline
\end{tabular}

generating halo-chaos and to achieve much better understand and control/suppression results on the complex halo-chaos phenomenon existing in high-intensity accelerator.

\section{Acknowledgements}

Project supported by the National Natural Science Foundation, Grants No. 19875080 and 70071047.

\section{References}

Ali, M.K. and Fang, J.Q. (1997) "Synchronization of chaos and hyperchaos using linear and nonlinear feedback function", Phys. Rev. E 55, 5285.

Chen, C. and Davidson, R.C. (1994) "Nonlinear resonances and chaotic behaviour in a periodic focused intense charged-particle beam", Phys. Rev. Lett. 72, 2195.

Chen, G. and Dong, X. (1998) From Chaos to Order: Methodologies, Perspectives and Applications (World Scientific, Singapore).

Chui, C.K. (1997) Wavelet: A Mathematical Tool for Signal Processing (SIAM, Philadelphia, PA).

Fang, J.Q. (1996) "Control and synchronization of chaos in nonlinear systems and prospects for applications (1)", Prog. Phys. 16(1), 1, (in Chinese).

Fang, J.Q. (1996) "Control and synchronization of chaos in nonlinear systems and prospects for applications (2)", Prog. Phys. 16(2), 137, in Chinese.

Fang, J.Q. (2000) "One of key problems for cleaner nuclear energy system driven by a high power accelerator", Nature 22(2), 63, in Chinese.

Fang, J.Q. and Ali, M.K. (1998) "Nonlinear feedback control of spatiotemporal chaos in coupled map lattices", Discrete Dyn. Nat. Soc. $1,283$.

Fang, J.Q. and Ali, M.K. (1999) "Control and synchronization of spatiotemporal chaos", In: Chen, G., ed, Control of Bifurcation and Chaos in Engineering Systems (CRC Press, New York), p 107.
Fang, J.-Q. and Chen, G. (2000) "Nonlinear feedback control of halochaos in high-intensity linacs", High Power Laser Particle Beams 12, $647-651$.

Fang, J.Q., Hong, Y.G. and Chen, G. (1999) "Switching manifold approach to chaos synchronization", Phys. Rev. E. 59, R2523.

Fang, J.-Q., Gao, Y., Weng, J.-Q., Chen, G. and Luo, X.-S. (2001) "Controlling beam halo-chaos using small wavelet feedback control method", Acta Phys. Sin. 50, 435-439.

Fang, J.-Q., Chen, G., Zhou, L.-L. and Huan, J.-J. (2001) "Physical mechanism of beam halo-chaos formation for high-current proton beam in a periodic-focusing channels an a nonlinear control strategy", Prog. Nat. Sci. 11, 6-15, also in Chinese edition, (11) (2001) pp. 113-120.

Fedotov, A.V. and Gluckstern, R.L. (1999) "Halo formation in threedimensional bunches with various phase space distributions", Phys. Rev. ST Accel. Beam ???2, 014201.

Fink, Y. and Chen, C. (1997) "Halo formation and chaos in root-meansquare matched beams propagating through a periodic solenoidal focusing channel", Phys. Rev. E 55, 7557.

Gluckstern, R.L. (1994) "Analytic model for halo formation in high current ion Linacs", Phys. Rev. Lett. 73(9), 1247.

Gluckstern, R.L. and Fedotov, A.V. (1999) "Coulomb scattering within a spherical beam bunch in a high current linear accelerator", Phys. Rev. ST Accel. Beam ???2, 054201.

Gluckstern, R.L., Fedotov, A.V., Kurennoy, S.S., et al., (1998) "Halo formation in three-dimensional bunches", Phys. Rev. E 58, 4977.

Lagniel, J.M. (1994) "On halo formation from space-charge dominated beams”, Nucl. Instrum. Methods Phys. Rev., A 345, 46.

Luo, Xiao-shu, Fang, Jin-Qing, Wang, Li-Hu, et al., (1999) "A new strategy of chaos control and a unified mechanism for several kinds of chaos control methods", Acta Phys. Sin. 8(12), 895-901.

Rubbia, C. A high gain energy amplifier operated with fast neutron, AIP Conference Proceedings 346, Third international conference on accelerator-driven transmutation technologies and applications, Las Vegas, July, 1994. (Also see CERN/AT/93-47(ET), 1993.)

Verhust, F. (1996) Nonlinear Differential Equations and Dynamical Systems, 2nd Ed. (Springer, Berlin), p 83.

Wang, F.T.S. (1999) "Particle-core study of halo dynamics in periodfocusing channels", Phys. Rev. E 61, 855-861. 


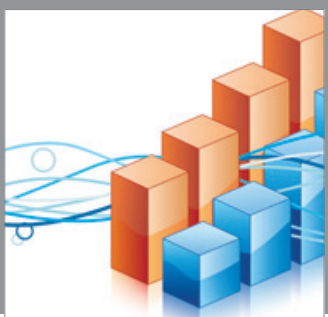

Advances in

Operations Research

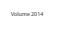

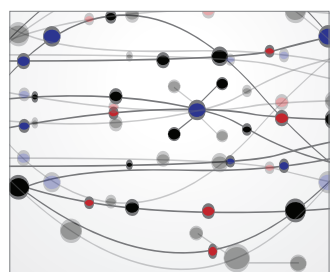

\section{The Scientific} World Journal
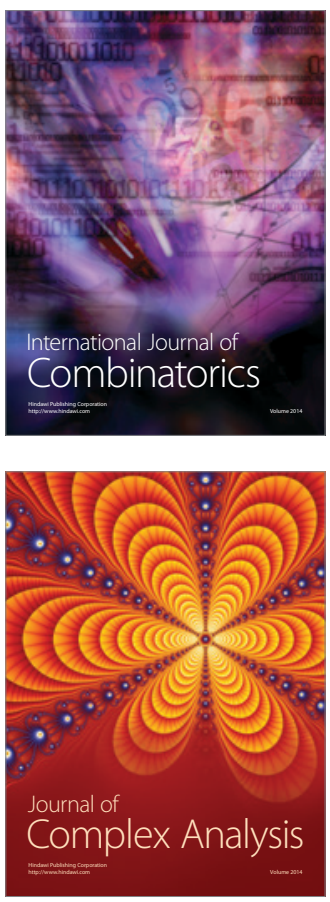

International Journal of

Mathematics and

Mathematical

Sciences
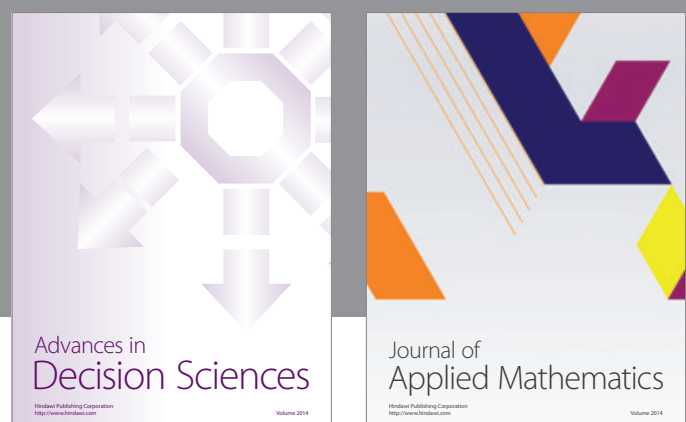

Journal of

Applied Mathematics
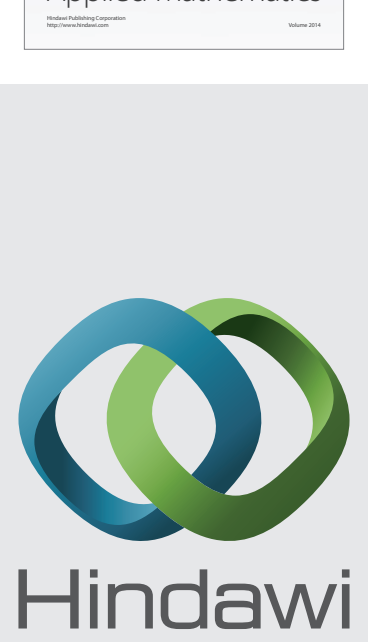

Submit your manuscripts at http://www.hindawi.com
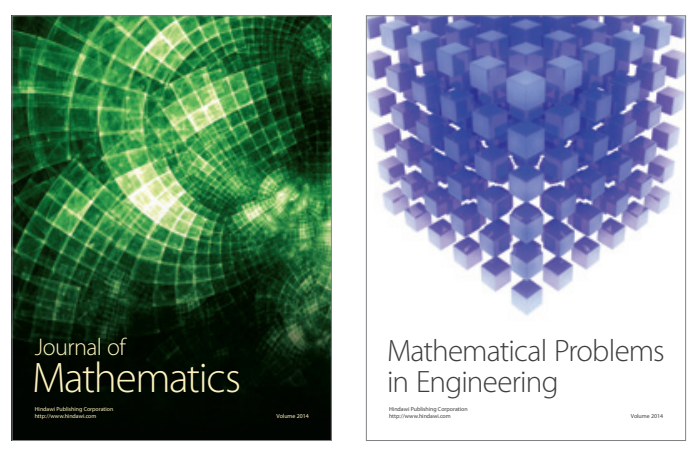

Mathematical Problems in Engineering
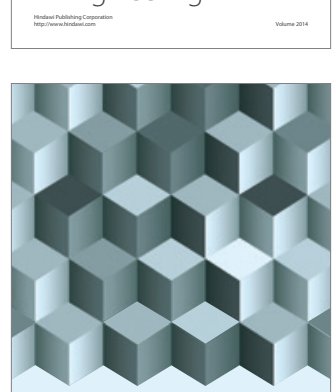

Journal of

Function Spaces
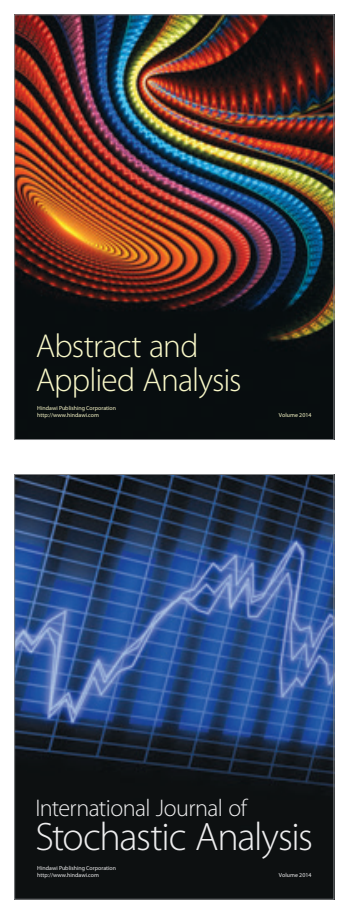

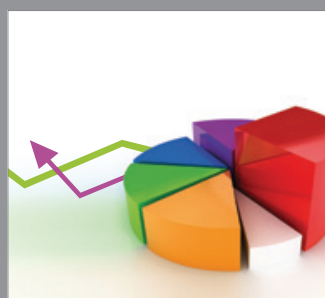

ournal of

Probability and Statistics

Promensencen
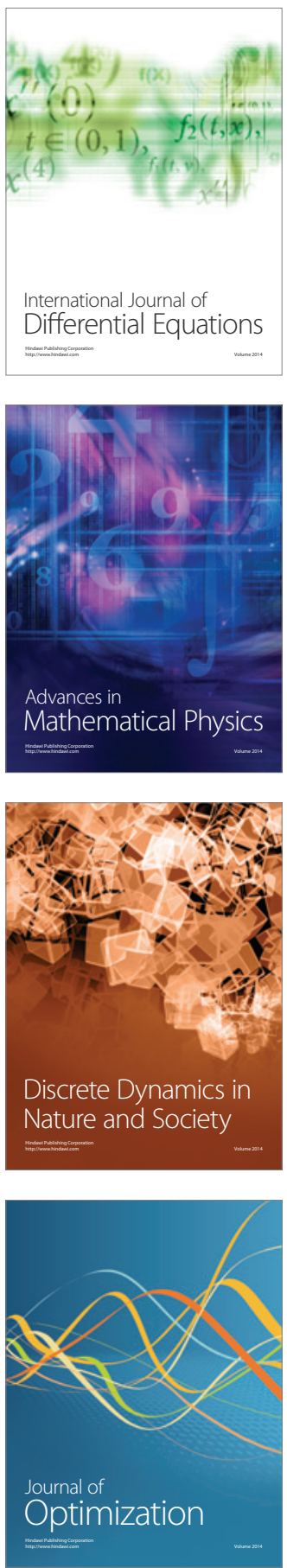